\title{
A Taxonomy of Motivational Affordances for Meaningful Gamified and Persuasive Technologies
}

\author{
Paul Weiser \\ Institute of Cartography \\ and Geoinformation \\ ETH Zurich \\ Stefano-Franscini-Platz 5 \\ 8093 Zurich, Switzerland \\ pweiser@ethz.ch
}

\author{
Dominik Bucher \\ Institute of Cartography \\ and Geoinformation \\ ETH Zurich \\ Stefano-Franscini-Platz 5 \\ 8093 Zurich, Switzerland \\ dobucher@ethz.ch
}

\author{
Francesca Cellina \\ Insitute for Applied Sustainability \\ to the Built Environment \\ SUPSI \\ Via Trevano \\ 6952 Canobbio, Switzerland \\ francesca.cellina@supsi.ch
}

\author{
Vanessa De Luca \\ Dep. of Environment \\ Constructions and Design \\ SUPSI \\ Via Trevano \\ 6952 Canobbio, Switzerland \\ vanessa.deluca@supsi.ch
}

\begin{abstract}
Sustainable human-computer interaction is investigating the role of persuasive and gamified technologies in encouraging people to engage in a more sustainable lifestyle. Motivation is a key requirement for behavior change, yet many persuasive systems do not sufficiently account for motivational aspects. In this paper we investigate under which circumstances components such as feedback and game elements (e.g., rewards) afford user motivation. The result is a taxonomy of design components that is grounded in well-established psychological theories on motivation. We illustrate how the taxonomy can contribute to the design of meaningful persuasive technologies by discussing a case study from the domain of sustainable mobility behavior (the project GoEco!).
\end{abstract}

\section{INTRODUCTION}

Research in the area of sustainable human-computer interaction (HCI) is investigating the role of persuasive technologies in encouraging people to engage in a more sustainable lifestyle (cf. [1], [2]). Studies have shown that information and communication technology (ICT) can indeed be effective in changing a person's habits, especially if one is already willing to change, but lacks a clear understanding of how the target behavior can be implemented ([3], [4]).

However, critics have also raised concerns about the limitations of current persuasive technologies (cf. [5], [6], [7]). For example, motivation is a key requirement for behavior change [8], yet many systems are not grounded in empirical research findings and theories on motivation. The result are applications that do not consider individual differences of their users, as well as offering few ways to engage in self-determined and collaborative approaches to behavior change ([9], [10]). Recent attempts to overcome these issues by harnessing the motivational power of games show promising first results (cf. [11]). However, in order to be truly meaningful, gamification ("the use of game design elements in non-game contexts" [12]) needs to tightly integrate a user's (motivational) needs and goals within an activity context (cf. [13], [14]).

In this paper, we investigate under which circumstances components typically employed by persuasive and gamified ICT (e.g., feedback and reward structures) afford user motivation. We present a taxonomy of motivational affordances (i.e., a classification of those properties that afford motivation [15]) constructed through linking components of persuasive and gamified systems to well-established theories on motivation and discuss it from the perspective of user and activity context. Using a case study from the domain of sustainable mobility behavior, we illustrate how our taxonomy can be used as a design guideline for persuasive and gamified information systems.

The remainder of this paper is structured as follows. We first review basic motivational needs (See Section III) and the requirements for behavior change (See Section IIII). In Section IV] we present our three-dimensional taxonomy of motivational affordances. In Section $\mathrm{V}$ we illustrate how the taxonomy can contribute to the design of a meaningful persuasive system. Section VI concludes this paper.

\section{Generic Motivational Needs}

Motivation "concerns those processes that give behavior its energy and direction" ([16], p. 22). Several scholars (cf. [5], [6], [7], [8], [15]) have argued that taking into account a user's motivational needs is one of the most crucial (but often neglected) design aspects for ICT.

\section{A. Psychological Needs}

Psychological needs are innate ([16], [17]) and encompass the desire for autonomy (choice), competence (skills) and relatedness (relationships).

1) Autonomy: The need for autonomy is the psychological desire to make self-determined choices, in particular during the initiation and regulation of behavior ([17], [16]). An example from ICT that fosters the need for autonomy is Khan Academy 1 It offers multiple paths (representing a series of lectures) that can lead to the same effect (e.g., understanding a concept or acquiring a skill). Thus, the user can make autonomous choices regarding which path to follow rather than being "forced" to follow one predetermined way to complete a lecture.

\footnotetext{
${ }^{1} \mathrm{~A}$ website offering free online education: http://www.khanacademy.org/
} 
2) Competence: Humans have the desire to become better at something, i.e., to acquire the skills necessary to perform a task efficiently [16]. Ideally, the process of skill acquisition keeps one in a state of "flow" (i.e., the complete absorption of one-self in a task [18]). This implies that a task should be neither too easy (boredom) nor too difficult (frustration) to complete. For example, many websites use what is called "onboarding" (cf. [19]), i.e., a tutorial phase during which the user is guided and instructed how to use the site and its features. Similarly, many computer games become more difficult as the player gets better in playing the game.

3) Relatedness: People have the need for engaging in relationships with others. Reeve [16] notes that "mere interaction" is a sufficient condition for invoking the feeling of relatedness. However, in order to satisfy the need for relatedness, additional mechanisms that signal recognition, acceptance and being valued are needed. In a number of studies, Fogg [20] showed that people can not only personally relate to artifacts but also appreciate and respond to them in ways that resemble humanhuman relationships. ICT has also the potential to leverage collective actions, during which an artifact acts either as a mediator between humans, or computers and humans work together on solving a particular problem (cf. [9], [10], [21]). A vivid example for a collaborative approach to problem solving is the rapid mapping of Haiti after it was hit by a 7.0 magnitude earthquake in 2010 2

\section{B. Social Needs}

Social needs are acquired through learning processes [16] and encompass the longing for achievement (do well), affiliation (approve and get approved) and intimacy (secure relationships), as well as leadership and followership (cf. [15]).

1) Achievement: Humans have the desire to do something well (against a norm) in order to show competence. Competitions of any form (with a task, oneself or others) can facilitate this need. For example, the website Stack Exchange $3^{3}$ gives its users (virtual) rewards for successfully answering a large number of questions.

2) Affiliation and Intimacy: Affiliation is the need to be approved by others, as well as make others happy and satisfied. For example, many social networking platforms provide mechanisms that allow to approve other people's content or get own content approved (cf. "likes"). Intimacy refers to the need for secure and rewarding relationships. For example, a popular online social network allows to mention the name of friends in a post, which can be interpreted as signaling (to others) a special form of relationship.

3) Leadership and Followership: Leadership refers to the desire to impact, control, and influence others [22]. Fogg [20] has shown that artifacts can exercise authority over humans, e.g., fictional characters that take on a teacher's role can encourage people to study harder. Because "there can be no

\footnotetext{
2 http://hot.openstreetmap.org/projects/haiti-2
}

3http://stackexchange.com/ leaders without followers" [23] there is also the desire for followership. For example, Researchgat ${ }^{4}$ uses both mechanisms. Power (thus leadership) is implicitly communicated through various metrics (e.g., someone's impact factor), while having many followers signals other people's interest in one's own work.

\section{REQUiREMENTS FOR BEHAVIOR CHANGE}

The previous section discussed innate (psychological) and learned (social) motivational needs. A system that intends to persuade its user to change behavior will likely be less effective, if it clearly violates universal motivational needs. However, needs only partially explain how motivation is generated and thus do not completely address how behavior can be changed.

Fogg [8] argued that whether or not some target behavior is performed depends on both a person's motivation and ability. In addition, there needs to be some form of trigger that prompts someone to actually perform the behavior. Fogg's model (See Figure 1p illustrates the following relationship between ability and motivation: If someone is highly motivated to do $\mathrm{x}$, she can be successful even if $\mathrm{x}$ is hard to do. However, if motivation is low, even something easy to do may become impossible. Triggers can either increase motivation (e.g., the prospect of winning a large sum of money) or ability (e.g., specific information on how to do $\mathrm{x}$ ).

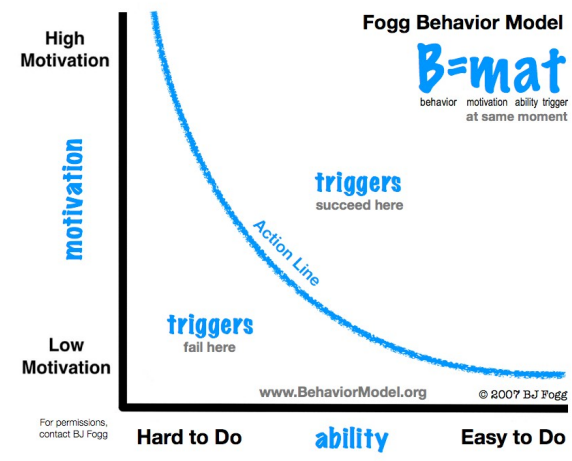

Fig. 1. Graphic illustration of the Fogg Behavior Model [8]. Retrieved from http://behaviormodel.org/

\section{A. Motivational Sources}

Behavior can either be extrinsically or intrinsically motivated. External motivation can be generated by environmental, social, or cultural events [16]. Intrinsic sources of motivation are related to a number of mental processes, in particular one's goals, expectations and the self.

Note, some of the elements found in persuasive and gamified systems can act as extrinsic motivators (e.g., receiving points for completing a task). Extrinsic motivators are discussed in detail in Section IV] In the following we only discuss intrinsic sources of motivation.

\footnotetext{
${ }^{4} \mathrm{~A}$ social network for scientists: http://www.researchgate.net
} 
1) Goals and Expectations: Goals are "internal representations of desired states", such as "outcomes, events, or processes" ([24], p. 338). One's individual goals can generate motivation and either energize or direct behavior. In addition, behavior is constantly guided through the evaluation of both efficacy ("ability to do x") and outcome ("likelihood that x succeeds") expectations in relation to some goal [16]. Both forms of expectation need to be reasonably high in order to feel motivated to do x (cf. [8]).

2) Attitudes, Beliefs, and Values: People's behavior is mainly reflected by their attitudes, beliefs, and values (cf. [25], [5]). Attitudes state what one likes or dislikes and can change relatively easy compared to beliefs and values. Beliefs are personal statements about "what is true and what is false". They are mostly based on experience and thus can change if one discovers new aspects of a topic related to a belief ([25], cf. [5], [26]). Values are behavioral ideals and preferences for experiences and form the core of our personality. They take the longest to change.

Change of attitudes, beliefs and values is often attained by having access to education (cf. [26]) or through revealing inconsistencies in one's cognitions. Humans have the strong desire to keep their behavior consistent with their beliefs. If one enters a state of inconsistency, e.g., by noticing that current behavior does not match current beliefs, behavior is adjusted to match the beliefs. Similarly, if current beliefs are noticed to not match some associated behavior, beliefs are adjusted to match behavior (cf. "cognitive dissonance" [27]).

3) The Self: The concept of self refers to the mental representation people have of themselves and is constructed through interactions with the environment, in particular through the feedback we receive from other people [28]. The self can influence motivation in two ways (cf. [16]). First, one strives to move from a present self towards an often idealized future self. Second, humans act in accordance with their conceptualization of one self, i.e., one behaves in such a way that properties of one's self are confirmed (cf. "confirmation bias" [29]).

\section{B. Ability}

Ability describes a user's capability to perform some behavior and is determined by her skills and the context. Context can be divided into environmental, technological, and individual context, the latter consisting of user characteristics, knowledge, preferences and situation ([30], pp. 214). Situation context includes the tasks and activities at hand, emotional state, physical capability as well as physiological condition or well-being ([30], [31]). Environmental context is locationdependent and includes factors such as the current weather, objects in proximity, or possibilities for interaction.

\section{Triggers}

A trigger is anything that prompts a user to actually perform a behavior. Fogg [8] termed triggers that act on a person's motivation sparks, and triggers that act on someone's ability facilitators. Sparks motivate by linking the target behavior to one of the motivational needs or by showing discrepancies between an unwanted behavior and one's attitudes, beliefs, values, or goals. For example, the suggestion to add more friends on Facebook can relate the behavior of logging in to the need for relatedness. Facilitators increase ability by providing knowledge (e.g., how to simplify a problem or by showing shortcuts that save time and money), as skill and context cannot be changed quickly. For example, informing someone about the opportunity to save money by going to a different gym on the way home (instead of taking the car to the regular gym later on), can make it easier for a user to reach the target behavior of reducing $\mathrm{CO}_{2}$ emissions [10]. A major challenge is to time a trigger effectively. In particular, triggers cannot succeed if the target behavior is far below the action line (See Figure 1).

\section{The Stages of Behavior Change}

Behavior change usually undergoes several stages, e.g., from a user's intention to change to actually performing and maintaining the behavior. $\mathrm{Li}$ et al. [32] distinguish between discovery and maintenance phase of behavior change (See Figure 33. During discovery, an individual wants to learn about the factors that influence behavior in order to be able to identify potential goals. In the maintenance phase a person actively strives towards the goals, until she goes back to discovery to discern more about herself and define new goals. Using the Transtheoretical Model [33], discovery and maintenance can further be broken down (See Figure 3 .

\section{TRANSTHEORETICAL MODEL}

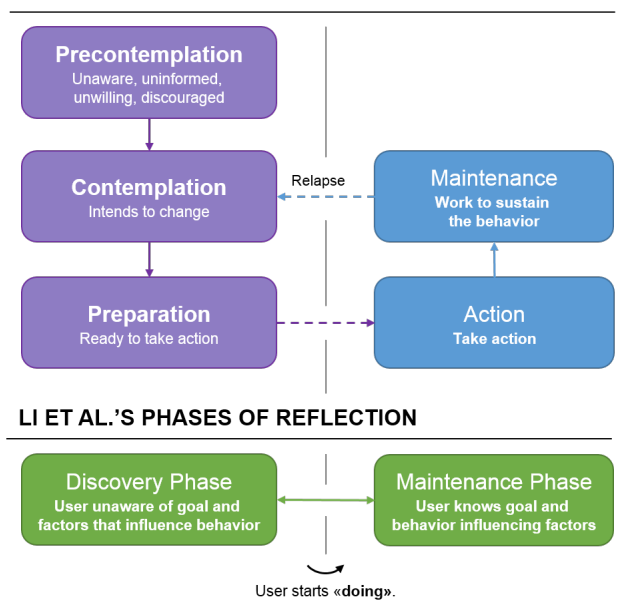

Fig. 2. Transtheoretical [33] vs. Li et al.'s [32] Behavior Change Models.

People in the discovery phase start in a state of precontemplation and have low motivation and ability. Instructional information or methods to raise awareness can help to move to the contemplation stage [5]. Continuous self-evaluation may eventually induce a transition into the preparation phase, during which someone starts developing a plan. During maintenance phase, individuals start performing actions. In carrying out the target behavior they may make a transition from novices to competent individuals and later from proficient individuals to experts (cf. [34]). Being experts, people have 
to be kept motivated (e.g., by not neglecting motivational needs or actively supporting ability and motivational sources) such that behavior is constantly performed ("rehearsed") and eventually internalized. However, the formation of habits can take a long time (cf. [35]). During the maintenance phase (if behavior has become a habit) people may relapse and fall back into the contemplation phase. Note, one can be in different phases for different types of behavior, e.g., precontemplating to change behavior A and preparing to change behavior B [5].

\section{TAXonomy of Motivational AfFordances}

In this section we discuss the components that persuasive and gamified technologies typically utilize to encourage behavior change from the perspective of motivational affordances (i.e., properties that afford user motivation [15]). To allow for meaningful gamified and persuasive systems, designers should choose components depending on both the objectives and the users of the system. To guide this process we present empirical evidence on the advantages and disadvantages of each component in terms of motivation given a specific user and activity context. We first outline general design principles and then present mechanics, i.e., possible means of interaction between system and user. Mechanics can be implemented using one or several concrete elements. For an overview of the taxonomy see Figure 3 .

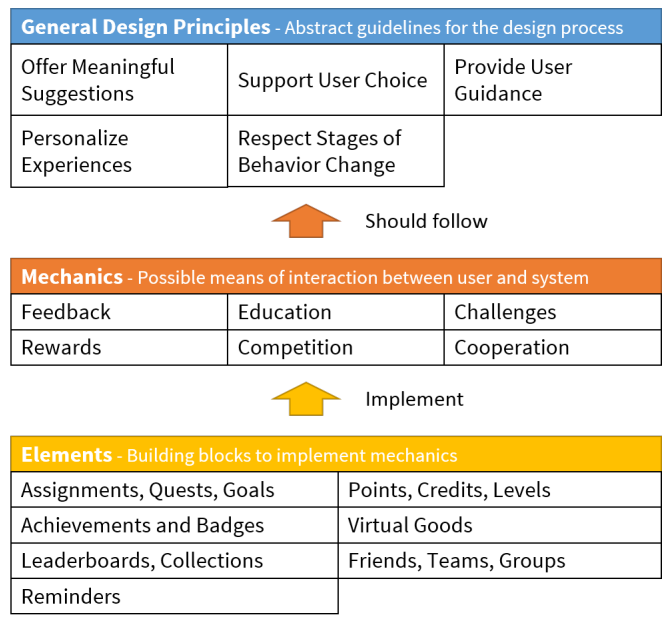

Fig. 3. Taxonomy of Motivational Affordances

\section{A. General Design Principles}

The following principles are abstract patterns and emerging properties that cannot be integrated in a system by simply adding one or several specific components. This section builds on the insights we gained from discussing the previous two sections on motivation.

1) Offer Meaningful Suggestions: There are two major challenges in designing persuasive systems that support people in pursuing their goals (e.g., to reduce their $\mathrm{CO}_{2}$ footprint). First, people need to become aware of behavior that is harmful to achieving their goal. Second, it is necessary to offer meaningful alternatives to current behavior if it does not align with the intended goal. For example, ICT can help to increase both efficacy and outcome expectations by making concrete suggestions on how behavior can be adjusted. However, suggestions can only be meaningful it they do not interfere with other goals one may have (cf. [36], [37], [38]). For example, the suggestion to take the bus instead of the car is not meaningful if at a given time no such option is available, or if it would imply to be be late for an important meeting. Meaningful suggestions need to integrate several sources of information, e.g., calendar and sensed transport mode. For a detailed discussion of such issues, see Weiser et al. [10].

2) Support User Choice: In order to support the need for autonomy, users should be given the ability to set their own goals (e.g., how to achieve some target behavior) and work on achieving goals at their own pace. Choices offered by the system need to go beyond "either-or" options (e.g., it should be tolerated that someone does not choose either one), in order to truly support one's psychological needs [39]. User may feel patronized by the system (cf. [6], [7]), if only one form of behavior is appropriate (i.e., the one the system designer had in mind). In addition, giving users autonomy over their actions enables user empowerment strategies [40]. For example, users may be given the possibility to explore causeand-effect relationships interactively and virtually rehearse behavior through means of simulation (cf. [20]).

3) Provide User Guidance: Users should be guided through the process of acquiring a skill necessary to perform the target behavior. For example, this may happen through task reduction and tunneling, i.e., making tasks simpler and controlling what a user experiences, respectively [20]. Clearly structured information can help to identify the desired outcome and support the user by suggesting how it can be achieved. Since people may fail (or choose not) to complete a particular task, the system needs to offer mechanisms that tolerate failure in order to avoid frustration [16]. Tolerance of failure implies a relaxed view on "appropriate behavior", thus strongly connecting skill acquisition (competence) to the need for autonomy.

4) Provide Personalized Experience: Designers should provide ways such that the users can express their self-identity. This can occur through tailoring content to a specific user (group) and mechanisms to let the users personalize their experience with the system ([15], [20]). However, it is difficult to categorize users without taking into account the particular domain context. Thus, useful personalized services will need to consider an iterative qualitative and quantitative design process (cf. [41], [42])

5) Design for Every Stage of Behavior Change: Information systems should provide behavior-related information that matches the requirements of the different stages of behavior change (see Section III-D). For example, during precontemplation the system should provide ways to collect, integrate, and reflect on behavior-related data (cf. [43]), such that the user is given the possibility to become aware of the "problematic" behavior. Information about easy ways to change behavior can increase a person's ability to change, while information about one's own or others' behavior can aid self-reflection. This may 
be followed by a person experiencing cognitive dissonance (e.g., if behavior does not match beliefs) or creating a spirit of competition resulting in higher levels of motivation. However, such effects are difficult to control. During the action phase, a system can provide informative feedback, e.g., alternatives to current behavior or suggestions to rearrange a task related to behavior change. Note, it is challenging to automatically recognize behavior change stages and to determine whether or not a change from one stage to the other has taken place. For a comprehensive discussion providing appropriate information during each behavior stage, see He et al. [5].

\section{B. Mechanics}

Mechanics are possible means of interaction between a user and the system.

1) Feedback: Feedback is visual, auditory, or haptic information on a user's current state, e.g., the currently performed behavior. Feedback is one of the most widely used mechanisms in persuasive and gamified systems (cf. [44], [45]). It can act as a motivational source by letting users evaluate how close current behavior is in relation to a set benchmark (i.e., goals, expectations and the self). Alternatively, feedback can facilitate user ability (e.g., providing suggestions about things that could have been optimized). Ideally, an information system that knows the current state and goal of a user can give feedback on the amount of discrepancy between current and goal state and offer meaningful suggestions on how to improve without patronizing the user (thus violating the need for autonomy). However, the ideal frequency and timeliness of feedback is notoriously difficult to determine ([5], [46]).

Feedback can either be given instantly or in an accumulated (offline) form. Instant feedback creates a stronger link between behavior and its consequences [45], however it should not interfere with the activity a user is currently performing. For example, because many countries prohibit cell phone use while operating a vehicle, feedback on driving behavior must only be given in a non-interfering way, e.g. in audible form or after driving (cf. [45]). Accumulated feedback and historical comparison can facilitate self-monitoring, supporting the process of becoming aware of one's behavior [47].

Other approaches to giving feedback aim at changing the representation of a task [37] or exploiting cognitive biases. For example, car drivers can be encouraged to slow down through unevenly spaced lines painted on the road. Driving at constant speed, a decreasing amount of line spacing creates a sensation of increasing driving speed (cf. [3]). Another approach mentioned by $\mathrm{He}$ et al. [5] is to provide encouragement even for small and unintentional behavior changes with the goal to motivate bigger and intentional future behavior. This works because performed behavior is justified by adjusting the belief system (cf. "cognitive dissonance"). On the other hand, it can be demotivating to receive feedback that contradicts aspects of one's own conceptualization because it introduces inconsistencies into one's mental representation (cf. [29]).

Sometimes feedback can backfire and generate a boomerang effect, i.e., generate behavior that is inverse to the intended behavior (cf. [48]). For example, telling someone that her behavior is above average usually leads to an adjustment of behavior towards this average, thus decreasing performance. In addition, one cannot assume that feedback on behavior is always used to actually change behavior (humans do not necessarily maximize utility [29]). However, perceived credibility of an artifact giving feedback (e.g., expertise, verifiability) can increase its persuasion capabilities [20].

2) User Education: Education is useful for situations in which one lacks the knowledge to achieve the intended target behavior, in particular, if it can provide advice on concrete tasks a user should perform [45]. Education can thus fulfill the need for competence and, up to some degree, the need for followership (e.g., if the device takes on a role as instructor, cf. [20], [49]). Similar to feedback, education can both facilitate user ability and generate motivation if it manages to activate mechanisms such as cognitive dissonance or foster competition. The primary and most effective use of education is in early stages of behavior change, where users are not fully aware of their problematic behavior, or lack the ability to change. In later stages, it can be used to continually supply novel and changing information to maintain interest [5]. Note, purely normative education ("you must (or must not) do x") have little effect on behavior change because it lacks contextual information ([40], [50]).

3) Challenges: A challenge is something (e.g., a task or problem) that is difficult to do. Challenges appeal to our psychological need for competence, because they can act as a benchmark against which we judge our performance. Challenges are good in settings with diverse users (cf. [51]), and with users that have no concrete goal (thus creating a goal) or don't know how to reach a goal (thus providing guidance) [49]. Challenges can facilitate user ability by splitting up a task into smaller and more manageable parts. Re-framing a challenge can decrease completion time and increase the likelihood it is completed. For example, this has been shown by restructuring a challenge that actually requires 8 steps to complete into a new challenge that formally consists of 10 steps, but gives away the first two steps for free (cf. [52]). Further, one may provide reasonable default goals (close to the intended target behavior), since most people do not deviate from default options even if they are given other choices [3].

4) Rewards: Rewards are anything that is given to users because of something they have achieved. Rewards are the prototypical form of external motivation [16], but can also fulfill the need for achievement and competence. For example, competence can be triggered when a user is rewarded for an increase in skill. Rewards can only generate motivation, because they don't change the ability to perform a behavior (except if the reward itself is a tool that increases ability).

Rewards only provide little motivation if they do not have a moment of surprise, e.g., occur in regular intervals and are predictable [53]. In addition, people who experience pleasure from receiving rewards will (over time) need bigger rewards to trigger the same amount of satisfaction [54]. Rewards can provide a strong motivational source, but like all extrinsic 
motivators, are less effective for changing behavior in the long run because externally motivated behavior only lasts as long as the external motivator exist [55]. Also, while rewards may invoke a feeling of achievement (through the act of collecting them), they can reduce motivation to do something to the act of collecting rewards (cf. [56]). On people who are intrinsically motivated to do something the presence of extrinsic motivators may have adverse effects [57].

Because of the negative aspects associated with rewards, they should be used with caution. Further research is still needed to identify methods that enable the internalization of externally motivated behavior (cf. [2]).

5) Competition and Comparison: Competition is the process during which rival parties attempt to achieve something, e.g., win a challenge. Competitions mainly appeal to our social need for achievement and leadership. They can increase motivation for people who are naturally competitive and have about the same skill, as well as are in comparable situations [58]. Competition can be problematic in settings where competition is unwanted, e.g., within a family [59], or between people who have different skill levels. If competitions are invoked through comparisons with one-self or peers, the results depend on the framing of the comparison (cf. [2]).

6) Cooperation: Cooperation is the collaborative process during which several parties attempt to achieve something (e.g., work together towards the solution of a problem). Cooperation appeals primarily to our need of relatedness, but can also satisfy the needs for affiliation (e.g. when a user gets accepted into a team) and leader- / followership (when cooperation involves some sort of leaders who direct and organize the cooperation). It is effective in settings where users are naturally social, know each other well, and have diverse levels of knowledge ([49], [58], [60]). In contrast, anonymous team cooperation seems to be less effective [61]. Cooperation can often complement competition because "intergroup competition leads individuals to enjoy an activity more than pure cooperation and pure competition" [62].

\section{Elements}

Design elements are concrete ways to implement the mechanics we discussed in the previous Section.

1) Assignments, Quests, and Goals: Assignments are concrete tasks a user needs to complete in order to complete a goal. "Forced" assignments may inhibit the user's need for autonomy and lead to users feeling patronized. Quests, i.e., optional challenges are thus better suited. Both quests and assignments can be "joyful" ways to break larger tasks in to smaller and more manageable parts (cf. [50]), thus supporting user competence. In order to support user autonomy, goals should be specifiable by the users themselves. Goals show better results on performed behavior when they are both specific and challenging [63]. This requires a solid understanding of how the target behavior can be achieved within a particular context ([64], [10]).

2) Points, Credits and Levels: Points and credits are numeric forms of rewards (i.e., of ratio type [38]) a user receives from the system (or other users) with the intention to score (rate) behavior. They also implicitly give feedback on user behavior. While points may represent a person's status and signal advancement towards a goal (cf. [65]), credits can usually be traded for other components (e.g., virtual goods see below) within the system. The amount of available points and credits needs to be adapted to the difficulty of the task involved, in order to account for different user personas and behavior stages (cf. [44]). Giving points for performing some behavior may have counter-intuitive effects. For example, people who are given points for making "green trips" tend to make unnecessary trips in order to score more points [44].

Sums of points or credits are often associated with levels (indications of an amount of something) and can communicate competence (i.e., invoke a feeling of progression). Levels can provide challenge, feedback, and reward at the same time. However, reaching levels too easily (or never) can make users question their usefulness (cf. [19]) and decrease motivation. The exact effects of points and levels on motivation are still unclear. For example, Mekler et al. [66] showed that they increased user engagement with a task, but did neither positively nor negatively affected autonomy, competence and intrinsic motivation.

3) Achievements and Badges: Achievements and badges are nominal forms of rewards [38] given for the completion of a task. Badges (visual representations of achievements) can serve various psychological functions [67]. They can act as a goal-setting device and thus challenge users to attain a goal and thus the badge. They can also act as instructors because they tell the user about possible actions within the system. Furthermore, they are indicators of reputation, thus can give indication about a user's level of engagement and her interests. In addition, they can act as status symbols, thus satisfy the need for leadership. Finally, they allow users to share experiences and thus foster group identification.

Online services that use badges show increased general and targeted (i.e., steering towards a badge) user activity. For example, Denny [68] showed a highly significant positive effect of badges (i.e., the possibility of getting them) on the quantity of contributions to an online learning Web site. At the same time, the quality of contributions was not negatively affected. In addition, badges may also encourage users to engage in a system in a more exploratory and experimental way (cf. [69], [70]). However, some users may not enjoy achievements at all, either because they generally don't see their purpose [71], or people perceive them as meaningless, e.g., in cases where getting them involves simple trial and error tasks. Achievements that involve contextual information (e.g., finding a location) are perceived as being more useful than random ones (cf. [72]).

4) Virtual Goods: Virtual goods are things that have some economic value and can be used for trading or display status and rank (cf. leadership). Some virtual goods may even be traded for real-world currency (cf. Gold farming ${ }^{5}$ ). Similar to

\footnotetext{
5 http://en.wikipedia.org/wiki/Gold_farming
} 
achievements and badges, acquiring goods can be a challenge and lead to competition and cooperation. It may create a strong relatedness to the system because material value is involved. However, if users intrinsically enjoy using the system, they show little interest in purchasing virtual goods. In addition, users' attitudes and beliefs about their peers attitudes strongly influence the willingness to purchase virtual goods [73]. Virtual goods share most of the benefits and disadvantages that are associated with other forms of rewards.

5) Leaderboards and Collections: Leaderboards (or highscore lists) and collections of rewards are public displays of rank and achievement. Leaderboards may appeal to our social needs to lead and follow. Both leaderboards and collections allow users to compare themselves with others, as well as selfevaluate performance (cf. [74]). Leaderboards can even have a demotivating effect on people who are lagging behind [75], e.g., users that just started to use the system (novices), but nevertheless need to compete with long-time users (experts). Methods to decrease the negative effects of leaderboards are to show them prominently only to users in the upper parts, show only positions of the leaderboard in close proximity to oneself, or to give more points to people lagging behind (cf. [76]).

6) Friends, Teams, and Groups: Friends, teams, and groups are elements found in many services to signal relatedness and intimacy with other people and can be part of cooperation and competition mechanics. They can be useful for diverse user bases, if leaders can help less advanced users in completing tasks. This may enable collaborative and collective actions towards the solution of a problem (cf. [9]). If users are too diverse to allow for direct forms of competition, forming teams and letting them compete against other teams can equalize individual differences. However, the emergence of "elite teams" may lead to demotivated and frustrated users (cf. [19]).

7) Reminders: Reminders can have both feedback and educational character. He et al. [5] suggested that reminders can help users to not relapse to earlier behavior stages or to encourage habit formation. However, even though reminders increase the repetition of behavior they apparently have little effect on habit formation [35]. Too frequent and regular reminders can become a nuisance, but even nagging reminders are sometimes perceived as positive [53]. In addition, social reminders (i.e., provided by peers) are more effective than automated computer reminders [77].

\section{Case Study: The Project GoEco!}

In order to illustrate how the previously discussed principles can be applied in a meaningful way, we use some context parameters of the project GoEco! (a collaboration between ETHZ and SUPSI in Switzerland). The goal of this short case study is to show how both user and activity context can guide the selection of concrete motivational affordances.

\section{A. Objectives}

GoEco! aims at understanding whether ICT-based ecofeedback information, social norms, and peer pressure can be effective in fostering changes in personal mobility behavior. In particular, we attempt to answer the question whether such a system can help to reduce private motorized transport and foster a transition to different mobility lifestyles, e.g., the increased use of vehicle-sharing or public transport. As such, the objectives are to significantly (1) reduce the use of private motorized transport without restricting individual mobility needs, and (2) limit overall mobility related energy consumption and $\mathrm{CO}_{2}$ emissions.

\section{B. Target Behavior}

The intended target behavior is people engaging in an energy-efficient mobility lifestyle as outlined in the objectives. However, a meaningful alignment of a person's daily routines and the system designer's intention to motivate people to engage in a sustainable lifestyle is challenging. This is because of the spatio-temporal and social constraints we experience daily, as well as the technical challenges involved in automatically recognizing and assessing behavior in order to give meaningful feedback. For example, the suggestion to take the bike to work is only meaningful, if it does not interfere with the need for being on time for a business meeting. Similarly, receiving a reward for taking the bus instead of the car can only be meaningful if the transport mode can be accurately be detected. For a detailed treatment of such issues see Weiser et al. [10].

\section{User Description}

For GoEco! a "living lab" experiment (i.e. a field study involving real-life users in real-world settings [78]) is set up, consisting of participants from two diverse geographic regions: (1) Ticino in Southern Switzerland, a vast area characterized by urban sprawl, and (2) the City of Zürich, a dense urban area offering a lot of alternative means of transport. The mobility patterns of users are tracked automatically using a mobile device. In order to perform reliable transport mode recognition (cf. [10]) and to provide meaningful suggestions, baseline data on each participant's behavior needs to be collected during an initial "training" phase.

We assume that users are initially in the discovery phase (See Section III-D). For example, someone contemplating change may be interested in receiving information on possible sustainable mobility options, because she lacks a clear understanding of how to implement the target behavior.

\section{Selected Mechanics and Elements}

We now briefly discuss how the insights gained in section IV can be used to determine if and how concrete mechanics and elements should be implemented given the context described above. In particular, the selection was guided by the intended target behavior of the users, the spatio-temporal constraints imposed by the geographic regions, and the empirical findings discussed in previous sections. However, our proposed solution should only be understood as an initial step in designing a meaningful gamified persuasive system and needs to be taken 
with a grain of salt. This is because it is based on theoretical considerations and not empirical findings that tested the particular configuration described above. Additional iterative design steps will be required, guided by the users' acceptance and perceived usefulness of the chosen components.

1) Education and Feedback: In our case study, we assume that potential users know about their "problematic" behavior and have an interest in becoming more sustainable. Thus, the system will not follow a normative approach, but instead focus on giving concrete information about current mobility behavior and possibilities for change based on the collected baseline data. Such information could include suggestions on using bus stops or car sharing possibilities. To support progression over the possible stages of behavior change, this information is shown more prominently when users are new to using the application. Later on, educative information is replaced by direct feedback on a user's behavior.

To help users to become more aware of the impacts of their mobility behavior we provide feedback mechanisms that are based on the continuous evaluation of their activities. Feedback is given in relation to the sensed transport mode. Instantaneous feedback is provided by default, except in cases where the system detects a transport mode that "prohibits" this form of feedback (e.g., while operating a vehicle). In such a case offline feedback, in the form of a summary, is given afterwards.

Feedback indicates how users progress towards a goal (either selected by the user or suggested by the system), but also includes meaningful suggestions on how to better align and connect daily activities. For example, suggestions such as (previously unknown) routes, modal choices or optimized daily schedules can increase the outcome expectations of users, as well as their knowledge and ability to perform behavior changes.

2) Challenges: Due to the geographic and personal context, users face a variety of mobility constraints (e.g., regarding modal options, or personal daily schedules) and thus may lack concrete goals for improving behavior. Because it is difficult to assess what kind of behavior improvement is feasible (cf. [10]), GoEco! only provides suggestions for goals that are inferred based on baseline data gathered during the initial "training" phase. In addition, goals also need to take into account contextual information, e.g., a user's daily schedule and possible spatio-temporal constraints. Users can then choose personal and meaningful goals in respect to the baseline modal choices, e.g.:

- "I will reduce the use of my car by $X$ percent."

$X$ specifies the quantitative target, to be chosen by the user. Because most people stick with defaults, a challenging value is provided based on the assessed feasibility of change.

While such goals may direct overall user behavior change, they don't facilitate a user's ability to reach them (i.e., they remain abstract in their goal description). With the intention to overcome this limitation, optional quests are generated, which one can complete in order to get closer to achieving goals. Quests can be understood as breaking abstract goals into smaller and more manageable parts in the form of concrete plans, e.g.:

- "Next week, on sunny days, I will go to work by bike." Thus, the quest "on sunny days..." could be part of the goal "reduce car usage by $x$ percent". Ideally, the difficulty of both goals and quests increases with user advancement. For example, if the system recognizes that a person is not able to complete a challenge, easier ones should be suggested. This supports progression along the stages of behavior change and ensures optimal challenge.

3) Rewards: The difficulty of a challenge should ideally be a function of user competence and context. For example, someone having public transport available in close vicinity can easily do without a car. For someone who lives in a small village in some rural part of Switzerland, however, this target behavior may be impossible. This observation may seem trivial, but again, it highlights the importance of taking contextual factors into account, if one wants to design a meaningful persuasive system.

Generally, rewards should be given in relation to the difficulty of a challenge. This allows users with different contexts and available options to compare themselves with each other. It also means that points given for the completion of challenges are not merely rewards but can be also used to generate competition mechanics (See below).

In order to provide additional optional goals and instruct users about possible actions within the system, GoEco! employs badges. However, they are only awarded for the completion of challenges potentially achievable by everyone. For example, someone who lives in a geographic region that provides no car-sharing services could never get the "Master of CarSharing" badge. To encourage exploratory behavior, badges are attributed for previously undisclosed actions. For example, if the system detects that a user went to the supermarket by bike instead of the regular car, a surprise badge is awarded. Finally, in an attempt to guide user activity, badges are also given for the recruitment of friends or sharing achievements on social media.

4) Competition: Competition mechanics are implemented using leaderboards that reflect a user's status using a point metric. In order to avoid some of the pitfalls of leaderboards, they are individualized for different user groups, i.e., leaderboards only show the performances of users with similar opportunities. Each user receives a separate ranking, in relation to the accessibility one has to alternative means of transport. In addition, top ranking users get to see leaderboards more prominently (to trigger achievement), while others actively have to request them (to avoid frustration).

5) Cooperation: In order to complement competition mechanics [62], GoEco! provides several ways of collaboration between users. For example, groups of users who already know each other can create teams to tackle challenges in a collaborative way. A team gets awarded points when individuals obtain good performances (compared to the overall norm) or help each other (e.g., by sharing a ride). To ensure that users who are part of a team are not favored (and thus could generate 
more points), team scores appear in a separate leaderboard and are completely orthogonal to individual scores.

\section{CONCLUSiOn AND OUTLOOK}

We presented a taxonomy of motivational affordances by linking design components typically found in persuasive technology to well-established theories on motivation. Using a case study from the domain of sustainable mobility behavior (the project GoEco!), we demonstrated how our taxonomy can be used as a design guideline for meaningful gamified persuasive systems.

In this paper, we did not explicitly address the many privacy and ethical concerns that arise from developing persuasive and location-aware technology. For example, while detailed and personalized information on people who commute the same route regularly could allow for a more accurate ride share service, it may also mean to give up traditional accounts of user privacy (cf. [79]).

While we were able to ground many of the discussed principles in existing literature, additional more conclusive studies on their properties in relation to user motivation are urgently needed. Existing empirical studies on gamified and persuasive systems often suffer from small sample sizes, the lack of control groups, and short timeframes ([11], [4]). Because the meaningful selection and implementation of motivational affordances depends strongly on contextual factors (e.g., the user base, in particular their needs and goals), future research should not only compare different affordances, but also their individual acceptance and usefulness in reaching defined objectives.

\section{ACKNOWLEDGMENT}

This research was supported by the Swiss Competence Center for Energy Research (SCCER) Efficient Technologies and Systems for Mobility and the Commission for Technology and Innovation (CTI). Additional support is acknowledged from the Swiss National Science Foundation (SNF).

\section{REFERENCES}

[1] C. DiSalvo, P. Sengers, and H. Brynjarsdóttir, "Mapping the landscape of sustainable hci," in Proceedings of the SIGCHI Conference on Human Factors in Computing Systems. ACM, 2010, pp. 1975-1984.

[2] J. E. Froehlich, "Gamifying green," in The Gameful World - Approaches, Issues, Applications, S. P. Walz and S. Deterding, Eds. MIT Press, 2015.

[3] R. H. Thaler and C. R. Sunstein, Nudge. Yale University Press, 2008.

[4] J. Hamari, J. Koivisto, and T. Pakkanen, "Do persuasive technologies persuade?-a review of empirical studies," in Persuasive Technology. Springer, 2014, pp. 118-136.

[5] H. A. He, S. Greenberg, and E. Huang, "One size does not fit all: applying the transtheoretical model to energy feedback technology design," Proceedings of the SIGCHI Conference on Human Factors in Computing Systems, pp. 927-936, 2010.

[6] H. Brynjarsdottir, M. Håkansson, J. Pierce, E. Baumer, C. DiSalvo, and P. Sengers, "Sustainably unpersuaded: how persuasion narrows our vision of sustainability," in Proceedings of the SIGCHI Conference on Human Factors in Computing Systems. ACM, 2012, pp. 947-956.

[7] M. Z. Huber and L. M. Hilty, "Gamification and sustainable consumption: overcoming the limitations of persuasive technologies," in ICT Innovations for Sustainability. Springer, 2015, pp. 367-385.

[8] B. J. Fogg, "A behavior model for persuasive design," in Proceedings of the 4th international Conference on Persuasive Technology. ACM, 2009 , p. 40
[9] H. J. Miller, "Beyond sharing: cultivating cooperative transportation systems through geographic information science," Journal of Transport Geography, vol. 31, pp. 296-308, 2013.

[10] P. Weiser, S. Scheider, D. Bucher, P. Kiefer, and M. Raubal, "Towards sustainable mobility behavior: Research challenges for location-aware information and communication technology," Geoinformatica, under review.

[11] J. Hamari, J. Koivisto, and H. Sarsa, "Does gamification work?-a literature review of empirical studies on gamification," in System Sciences (HICSS), 2014 47th Hawaii International Conference on. IEEE, 2014, pp. 3025-3034.

[12] S. Deterding, D. Dixon, R. Khaled, and L. Nacke, "From game design elements to gamefulness: defining gamification," in Proceedings of the 15th International Academic MindTrek Conference: Envisioning Future Media Environments. ACM, 2011, pp. 9-15.

[13] S. Deterding, "Situated motivational affordances of game elements: A conceptual model," in Gamification: Using Game Design Elements in Non-Gaming Contexts, a workshop at CHI, 2011.

[14] S. Nicholson, "A user-centered theoretical framework for meaningful gamification," in Games+ Learning+ Society, 2012.

[15] P. Zhang, "Toward a positive design theory: principles for designing motivating information and communication technology," Designing Information and Organizations with a Positive Lens: a volume of the Advances in Appreciative Inquiry series, Amsterdam: Elsevier, pp. 4573, 2008.

[16] J. Reeve and J. Reeve, Understanding motivation and emotion. Wiley New York, 2001.

[17] E. L. Deci and R. M. Ryan, Handbook of self-determination research. University Rochester Press, 2002.

[18] M. Csikszentmihalyi, "Flow," in Flow and the Foundations of Positive Psychology. Springer Netherlands, 2014, pp. 227-238.

[19] K. Werbach and D. Hunter, For the win: How game thinking can revolutionize your business. Wharton Digital Press, 2012.

[20] B. J. Fogg, Persuasive technology: using computers to change what we think and do. Morgan Kaufmann, 2002.

[21] N. R. Jennings, L. Moreau, D. Nicholson, S. D. Ramchurn, S. Roberts, T. Rodden, and A. Rogers, "Human-agent collectives," Communications of the ACM, vol. 57, no. 12, 2014.

[22] D. G. Winter, The power motive. Free Press New York, 1973

[23] R. Goffee and G. Jones, "Followership: Its personal too," Harvard Business Review, vol. 79, no. 11, p. 148, 2001.

[24] J. T. Austin and J. B. Vancouver, "Goal constructs in psychology: Structure, process, and content." Psychological bulletin, vol. 120, no. 3, p. 338, 1996.

[25] S. A. Beebe, S. J. Beebe, and M. V. Redmond, "Interpersonal communication: Relating to others," 2010.

[26] M. Rokeach et al., The nature of human values, vol. 438

[27] L. Festinger, A theory of cognitive dissonance. Stanford university press, 1962, vol. 2.

[28] H. Markus, "Self-knowledge: An expanded view," Journal of personality, vol. 51, no. 3, pp. 543-565, 1983.

[29] D. Kahneman, Thinking, fast and slow. Macmillan, 2011.

[30] A. Brimicombe and C. Li, Location-based services and geo-information engineering. John Wiley \& Sons, 2009, vol. 21.

[31] S. Consolvo, D. W. McDonald, and J. a. Landay, "Theory-driven design strategies for technologies that support behavior change in everyday life," Proceedings of the 27th international conference on Human factors in computing systems - CHI 09, pp. 405-414, 2009.

[32] I. Li, A. Dey, and J. Forlizzi, "Understanding My Data, Myself: Supporting Self-Reflection with Ubicomp Technologies," Proceedings of the 13th international conference on Ubiquitous computing, pp. 405414, 2011.

[33] J. O. Prochaska and W. F. Velicer, "The Transtheoretical Change Model of Health Behavior," American Journal of Health Promotion, vol. 12, pp. 38-48, 1997.

[34] S. E. Dreyfuss and H. L. Dreyfus, "A five-stage model of the mental activities involved in directed skill acquisition," Operations Research Center, no. February, pp. 1-18, 1980.

[35] K. Stawarz, A. L. Cox, and A. Blandford, "Beyond self-tracking and reminders: Designing smartphone apps that support habit formation," Proc. CHI 2015, 2015.

[36] A. Abdalla, P. Weiser, and A. U. Frank, "Design principles for spatiotemporally enabled pim tools: A qualitative analysis of trip planning," 
in Geographic Information Science at the Heart of Europe. Springer International Publishing, 2013, pp. 323-336.

[37] M. Hassenzahl and M. Laschke, "Pleasurable troublemakers," The Gameful World: Approaches, Issues, Applications, p. 167, 2015.

[38] S. Scheider, P. Kiefer, P. Weiser, M. Raubal, and C. Sailer, "Score design for meaningful gamification," in Researching Gamification: Strategies, Opportunities, Challenges, Ethics - a workshop at CHI, 2015.

[39] J. Reeve, G. Nix, and D. Hamm, "Testing models of the experience of self-determination in intrinsic motivation and the conundrum of choice." Journal of educational psychology, vol. 95, no. 2, p. 375, 2003.

[40] P. A. Tengland, "Behavior change or empowerment: On the ethics of health-promotion strategies," Public Health Ethics, vol. 5, no. 2, pp. 140-153, 2012.

[41] D. Dixon, "Player types and gamification," Proceedings of the CHI 2011 Workshop on Gamification, 2011.

[42] A. Canossa and A. Drachen, "Patterns of play: Play-personas in usercentred game development," in Breaking New Ground: Innovation in Games, Play, Practice and Theory: Proceedings of the 2009 DiGRA Conference, London, Brunel University (September 2009), 2009.

[43] I. Li, A. Dey, and J. Forlizzi, "A stage-based model of personal informatics systems," Proceedings of the 28th international conference on Human factors in computing systems CHI 10, p. 557, 2010.

[44] J. Froehlich, T. Dillahunt, P. Klasnja, J. Mankoff, S. Consolvo, B. Harrison, and J. A. Landay, "Ubigreen: Investigating a mobile tool for tracking and supporting green transportation habits."

[45] J. Tulusan, L. Soi, J. Paefgen, M. Brogle, and T. Staake, "Which ecofeedback types prefer drivers most ?" pp. 1-8, 2011.

[46] J. Froehlich, L. Findlater, and J. Landay, "The design of eco-feedback technology," in Proceedings of the SIGCHI Conference on Human Factors in Computing Systems. ACM, 2010, pp. 1999-2008.

[47] R. K. Jain, J. E. Taylor, and G. Peschiera, "Assessing eco-feedback interface usage and design to drive energy efficiency in buildings," Energy and Buildings, vol. 48, pp. 8-17, 2012.

[48] R. B. Cialdini, "Crafting normative messages to protect the environment," Current directions in psychological science, vol. 12, no. 4, pp. 105-109, 2003.

[49] A. Gustafsson, C. Katzeff, and M. Bang, "Evaluation of a pervasive game for domestic energy engagement among teenagers," Computers in Entertainment (CIE), vol. 7, no. 4, p. 54, 2009.

[50] C. Fischer, "Feedback on household electricity consumption: A tool for saving energy?" Energy Efficiency, vol. 1, pp. 79-104, 2008.

[51] a. Jylhä, P. Nurmi, M. Sirén, S. Hemminki, and G. Jacucci, "Matkahupi: A persuasive mobile application for sustainable mobility," UbiComp 2013 Adjunct - Adjunct Publication of the 2013 ACM Conference on Ubiquitous Computing, pp. 227-230, 2013.

[52] J. C. Nunes and X. Drèze, "The endowed progress effect: How artificial advancement increases effort," Journal of Consumer Research, vol. 32, no. 4, pp. 504-512, 2006.

[53] S. Munson and S. Consolvo, "Exploring Goal-setting, Rewards, Selfmonitoring, and Sharing to Motivate Physical Activity," International Conference on Pervasive Computing Technologies for Healthcare, pp. 25-32, 2012.

[54] S. Frederick and G. Loewenstein, "Hedonic adaptation," in Well-being: The foundations of hedonic psychology, D. Kahneman, E. Diener, and N. Schwarz, Eds. Russel Sage Foundation, 1999.

[55] E. L. Deci, G. Betley, J. Kahle, L. Abrams, and J. Porac, "When Trying To Win: Competition and Intrinsic Motivation," Personality and Social Psychology Bulletin1, vol. 7, no. 1, pp. 79-83, 1981.

[56] M. Laschke and M. Hassenzahl, "Mayor or patron? the difference between a badge and a meaningful story," $C H I E A^{\prime} 11,2011$.

[57] E. L. Deci, R. Koestner, and R. M. Ryan, "A meta-analytic review of experiments examining the effects of extrinsic rewards on intrinsic motivation.” Psychological bulletin, vol. 125, no. 6, p. 627, 1999.

[58] S. Sepehr and M. Head, "Competition as an element of gamification for learning: An exploratory longitudinal investigation," in Proceedings of the First International Conference on Gameful Design, Research, and Applications, ser. Gamification '13. New York, NY, USA: ACM, 2013, pp. 2-9.

[59] J. Froehlich, L. Findlater, M. Ostergren, S. Ramanathan, J. Peterson, I. Wragg, E. Larson, F. Fu, M. Bai, S. Patel, and J. a. Landay, "The design and evaluation of prototype eco-feedback displays for fixturelevel water usage data," Proceedings of the SIGCHI Conference on Human Factors in Computing Systems, pp. 2367-2376, 2012.
[60] J. H. Jung, C. Schneider, and J. Valacich, "Enhancing the Motivational Affordance of Information Systems: The Effects of Real-Time Performance Feedback and Goal Setting in Group Collaboration Environments," Management Science, vol. 56, no. January 2015, pp. 724-742, 2010.

[61] J. J. Lin, L. Mamykina, S. Lindtner, G. Delajoux, and H. B. Strub, "FishnSteps: Encouraging Physical Activity with an Interactive Computer Game," UbiComp 2006: Ubiquitous Computing, pp. 261-278, 2006.

[62] J. M. Tauer and J. M. Harackiewicz, "The effects of cooperation and competition on intrinsic motivation and performance." Journal of personality and social psychology, vol. 86, no. 6, p. 849, 2004.

[63] E. A. Locke and G. P. Latham, "Building a practically useful theory of goal setting and task motivation: A 35-year odyssey." American psychologist, vol. 57, no. 9, p. 705, 2002.

[64] P. Saini and J. Lacroix, "Self-setting of physical activity goals and effects on perceived difficulty, importance and competence," in Proceedings of the 4th International Conference on Persuasive Technology. ACM, 2009 , p. 33.

[65] J. P. Zagal, M. Mateas, C. Fernández-Vara, B. Hochhalter, and N. Lichti, "Towards an ontolqgical language for game analysis," Worlds in Play: International Perspectives on Digital Games Research, vol. 21, p. 21, 2007.

[66] E. D. Mekler, F. Brühlmann, K. Opwis, and A. N. Tuch, "Do points, levels and leaderboards harm intrinsic motivation?: an empirical analysis of common gamification elements," in Proceedings of the First International Conference on Gameful Design, Research, and Applications. ACM, 2013, pp. 66-73.

[67] J. Antin and E. F. Churchill, "Badges in social media: A social psychological perspective," in CHI 2011 Gamification Workshop Proceedings (Vancouver, BC, Canada, 2011), 2011.

[68] P. Denny, "The effect of virtual achievements on student engagement," in Proceedings of the SIGCHI Conference on Human Factors in Computing Systems. ACM, 2013, pp. 763-772.

[69] A. Anderson, D. Huttenlocher, J. Kleinberg, and J. Leskovec, "Steering user behavior with badges," in Proceedings of the 22nd international conference on World Wide Web. International World Wide Web Conferences Steering Committee, 2013, pp. 95-106.

[70] J. Hamari, "Do badges increase user activity? a field experiment on the effects of gamification," Computers in Human Behavior, 2015.

[71] M. Montola, T. Nummenmaa, A. Lucero, M. Boberg, and H. Korhonen, "Applying game achievement systems to enhance user experience in a photo sharing service," in Proceedings of the 13th International MindTrek Conference: Everyday Life in the Ubiquitous Era. ACM, 2009, pp. 94-97.

[72] Z. Fitz-Walter, D. Tjondronegoro, and P. Wyeth, "Orientation passport: using gamification to engage university students," in Proceedings of the 23rd Australian Computer-Human Interaction Conference. ACM, 2011, pp. $122-125$.

[73] J. Hamari, "Why do people buy virtual goods? attitude towards virtual good purchases versus game enjoyment," International Journal of Information Management, vol. 35, pp. 299-308, 2015.

[74] J. P. Costa, R. R. Wehbe, J. Robb, and L. E. Nacke, "Time's up: studying leaderboards for engaging punctual behaviour," in Proceedings of the First International Conference on Gameful Design, Research, and Applications. ACM, 2013, pp. 26-33.

[75] M. Oliveira and S. Petersen, "Co-design of neighbourhood services using gamification cards," in HCI in Business. Springer, 2014, pp. 419-428.

[76] J. E. Cechanowicz, C. Gutwin, S. Bateman, R. Mandryk, and I. Stavness, "Improving player balancing in racing games," in Proceedings of the first ACM SIGCHI annual symposium on Computer-human interaction in play. ACM, 2014, pp. 47-56.

[77] M.-C. Chiu, S.-P. Chang, Y.-C. Chang, H.-H. Chu, C. C.-H. Chen, F.-H. Hsiao, and J.-C. Ko, "Playful bottle: a mobile social persuasion system to motivate healthy water intake," in Proceedings of the 11th international conference on Ubiquitous computing. ACM, 2009, pp. 185-194.

[78] A. Higgins and S. Klein, "Introduction to the living lab approach," in Accelerating Global Supply Chains with IT-Innovation, Y.-H. Tan and et al., Eds. Berlin Heidelberg: Springer-Verlag, 2011.

[79] P. Weiser and S. Scheider, "A civilized cyberspace for geoprivacy," in Proceedings of the 1st ACM SIGSPATIAL International Workshop on Privacy in Geographic Information Collection and Analysis. ACM, 2014, p. 5. 